\title{
Anthropometric characteristics, quality of life and functional capacity in adults living with HIV in Puerto Rico
}

\author{
Christian J. Rivera Ruiz a, Farah A. Ramirez-Marrero a,b, Martin G. Rosario c, \\ a University of Puerto Rico, Rio Piedras Campus \\ b University of Puerto Rico, Medical Sciences Campus \\ c Physical Therapy Program, Texas Woman's University, Dallas Campus; 5500 Southwestern Medical Ave. Dallas, TX 75235- \\ 7299, United States. \\ *Corresponding author Email: mrosario1@twu.edu \\ DOI: https://doi.org/10.34256/ijpefs21312
}

Received: 28-08-2021, Revised: 23-09-2021; Accepted: 25-09-2021; Published: 30-09-2021

\begin{abstract}
Survival and longevity rates in people living with HIV (HIV+) have increased with the availability and use of antiretroviral therapy (ART). However, despite the above HIV+ adults treated with ART have a higher risk of developing dyslipidemia and high waist circumference. In addition, they have lower cardiorespiratory fitness, loss of muscle mass, reduced balance, and reduced functional capacity, which affects their quality of life. To explore the impact of balance perception, treadmill time, grip strength, body mass index (BMI), and waist-to-height ratio (WHtR) on quality of life in Latino Hispanic people living with HIV. This study recruited twenty-five participants from a community-based center, La Perla de Gran Precio, in San Juan, Puerto Rico, with the medical diagnosis of HIV. Descriptive measures were obtained for all variables of interest, and correlation and regression analyses were used to assess the associations between functional capacity, quality of life, and anthropometric measures. Result: Men had greater left- and right-hand grip strength than women $(86.9 \pm 18.8,56.9 \pm 26.8 \mathrm{~kg} ; \mathrm{p}=0.003$ and $87.6 \pm 15.1$ vs. $61.4 \pm 26.6 \mathrm{~kg} ; \mathrm{p}=0.004)$. Two anthropometric variables showed a trend toward a moderate positive correlation with quality of life: WHtR $(r=-0.38, p=0.12)$ and BMI $(r=-0.38, p=p-0.14)$. Although gender differences in upper body strength are expected, handgrip strength is within the gender-specific average range of the general population. The integration of anthropometric characteristics and upper body strength when prescribing exercise must be considered since these factors influence functional capacity and quality of life among HIV+ adults.
\end{abstract}

Keywords: HIV, Functional Capacity, Anthropometrics, Quality of Life

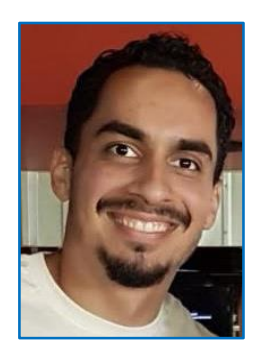

Christian J. Rivera Ruiz, MExSC Obtained his Master degree in exercise sciences with concentration in exercise physiology from the University of Puerto Rico Rio Piedras Campus. Bachelor in Physical education from University of Puerto Rico Rio Piedras Campus. Former high school physical education teacher and fitness instructor at an HIV community clinic La Perla de Gran Precio in San Juan, Puerto Rico.

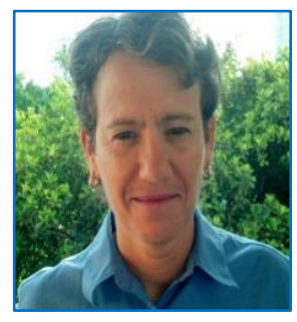

Dr. Farah A. Ramírez-Marrero is an exercise physiologist graduated from The Ohio State University, with two post-doctorate trainings in clinical research completed at the University of Puerto Rico Medical School and Mayo Clinic in Rochester, MN. She is a full professor at the University of Puerto Rico Río Piedras Campus where she teaches undergraduate and graduate courses in exercise physiology, research methods, exercise prescription, among others. She also conducts clinical research at the University of Puerto Rico Rio Piedras Campus and Medical Sciences Campus where she is in charge of a clinical exercise physiology laboratory located in the AIDS Clinical Trials Unit (project ACTU). She directs and collaborates with various research projects including healthy children and adults, as well as adult with chronic disease such as those living with HIV infection.

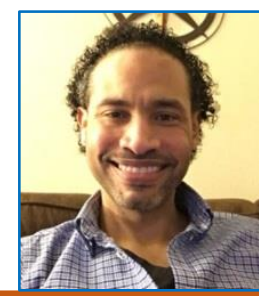

Martin G. Rosario PT, Ph.D., CSFI, ATRIC. Obtained his Ph.D. in Neuroanatomy from the University of Puerto Rico Medical Sciences Campus. Master in Physical Therapy from the University of Puerto Rico Medical 
Sciences Campus School of Physical Therapy. Bachelor in Biology with a Major in Human Focus from University of Puerto Rico Bayamon Campus. Currently serves as an Assistant Professor at Texas Woman's University (TWU) School of Physical Therapy Dallas, Clinical Researcher at an HIV community clinic La Perla de Gran Precio in Puerto Rico, and Director/Coordinator of Motor Control Research Laboratory at TWU Dallas.

\section{Introduction}

Infection with the human immunodeficiency virus (HIV) is a growing worldwide epidemic, with an infection prevalence near 37.9 million by 2018, of which approximately 1.7 million were newly infected [1]. In 2017, the Spanish-speaking community reached $26 \%(9,889)$ of 38,739 recent cases diagnosed in the United States and its territories [2]. By 2017, Puerto Rico had the fifth-highest prevalence rate of HIV infections in the United States and its territories, with 16,809 per 100,000 people [3].

Survival rates and longevity among HIV+ adults have expanded because of the availability and use of antiretroviral therapy (ART). However, HIV+ adults treated with ART are at significant cardiometabolic risk [4]. Based on the above, those living with HIV can experience various consequences, such as elevated waist circumference, dyslipidemia (elevated triglycerides, and low high-density lipoprotein (HDL) [5]. In addition, health-related quality of life [6] can be affected due to lower cardiorespiratory fitness [5], weight loss [7], balance instability [8], risk of falls during daily tasks [9], and a reduction in functional capacity. Functional capacity defines the competence of a person to perform physical activities independently, and a reduction in functional capacity has been associated with decreased quality of life, morbidity, and premature mortality among HIV+ adults [10].

Assessment of functional capacity in HIV+ adults is critical for the proper development and implementation of exercise prescriptions that could benefit overall health in this population. Sub-maximal tests of cardiorespiratory fitness and balance tests have been used and are generally accepted as functional capacity measures [11]. However, with limited consensus on the most appropriate steps to determine functional capacity, published studies have suggested a direct association between functional capacity and improved quality of life [12]. Based on the above, in those living with HIV in Puerto Rico, the current study intends to identify associations between functional capacity, anthropometric characteristics, and quality of life.

\section{Methods}

\subsection{Study design}

This descriptive cross-sectional study was conducted at the" LPGP community-based health and alternative medicine center in San Juan, Puerto Rico. LPGP provides various services for HIV+ adults and is funded by the United States Department of Health and Human Services (USDHHS) through the Ryan White program. This center provides a structured exercise program and physical therapy services for low-income HIV+ adults as part of the alternative medicine services. After referral by an immunologist, participants were evaluated by a licensed physical therapy professional and referred to a physical fitness instructor who conducts fitness assessments and prepares a prescription and exercise plan in consultation with the physical therapist.

\subsection{Participants}

A group of $25 \mathrm{HIV}+$ adults (15 men and 10 women) participants at LPGP who qualified for the study and had completed quality of life, anthropometric, and fitness evaluations as indicators of functional capacity at LPGP volunteered for this study. After detailed information on the risks and benefits of participation was provided, and all questions were answered, each participant signed an informed consent previously approved by the LPGP organization and the Texas Woman's University (Dallas, TX) Institutional Review Board (IRB). Then, secondary data analysis was conducted using the information from each participant's clinical records at the LPGP.

\subsection{Eligibility criteria}

This study excluded participants in the LPGP with AIDS diagnosis, trauma, or injuries that potentially hindered or altered their physical fitness tests.

\subsection{Intervention}

Functional capacity. A submaximal progressive load treadmill test using the Ross protocol was used to evaluate the functional capacity. This protocol allows the estimation of cardiorespiratory fitness based on the total time of assessment completion. The Ross protocol 
showed excellent concordance and reliability ( $R 2=0.85)$ compared to the Astrand protocol [11]; therefore, it is considered an efficient and effective cardiorespiratory fitness test.

Cardiorespiratory fitness evaluation started with a 3-minute warm-up beginning at $2.0 \mathrm{mph}$ and increasing the speed by $0.5 \mathrm{mph}$ each minute while maintaining the incline at $0 \%$. After the warm-up, the submaximal ROSS protocol was initiated. The speed was kept constant at $3.4 \mathrm{mph}$, and the slope was increased by $3 \%$ every $3 \mathrm{~min}$. The test ended when participants indicated that they could no longer continue. The reasons for stopping the test included lower extremity pain, muscle fatigue, or fear of losing balance.

Isometric grip strength is another fitness test that is used as an indicator of functional capacity. This test has been significantly associated with low functional disability in men and women aged $>50$ [13] and has adequately predicted the risk of premature mortality [14]. It is also regularly used in studies on HIV+ adults [15]. With a handheld dynamometer (JAMAR hydraulic hand dynamometer, JLW Instruments, Chicago, IL, USA), each participant was instructed to grab and hold for 30 seconds. The test was repeated three times with a period of one min of rest between attempts, and the maximum value of the three attempts was registered as the maximal isometric grip strength in pounds.

The assessment of balance perception using the Activities-Specific Balance Confidence Scale (ABC) is another commonly used functional capacity criterion. This 16-item questionnaire predicts falls in older adults [16] and determines balance disturbance in everyday activities among HIV+ Latinos-Hispanics [17]. With the $A B C$ scale, participants indicated their confidence level (between-0-100) in performing different tasks without losing balance ( $0 \%$ meant distrustful, and $100 \%$ meant total confidence in achieving the task). With a total score obtained by calculating the average of all percentages given to each activity, this questionnaire's outcome reflects the subject's perception of performing actions that involve balance.

Anthropometric Characteristics: Taking both while the participants were barefooted, weight was measured using the Baseline scale (model 12-1190, Fabrication Enterprises Inc., White Plains, NY) and height with a Detecto stadiometer (model 3P7044, Cardinal Scale Manufacturing Co., Webb City, MO). Body mass index (BMI) in $\mathrm{kg} / \mathrm{m}^{2}$ was calculated. Waist circumference was measured using a Gulick Baseline anthropometric tape (model 12-1201, Fabrication Enterprises Inc., White Plains, NY) placed horizontally at the narrowest part of the abdominal area between the lower rib and the iliac crest. Waist circumference and height were used to calculate the waist-to-height ratio (WHtR).

Quality of Life. The "Functional Assessment of Human Immunodeficiency Virus Infection" (FAHI) questionnaire was used to determine life quality. This 47-item questionnaire includes dimensions such as the perception of general physical health, emotional state, general state of functioning, social environment, and mental activity [18]. From a list of statements, each participant chose a number between 0 and 4 (nothing, a little, a lot, or very much) corresponding to each item's importance and applicability to their current life situation. When the questionnaire was completed, each subscale score was added to obtain the total value. A high FAHI score translates into a good or adequate quality of life. In addition, the FAHI questionnaire has demonstrated acceptable psychometric properties and can distinguish health status modifications among HIV+ adults [18].

Statistical Analysis. Analyses of the central tendency and variability of all variables of interest were completed. In addition, independent Student's t-tests were used to detect differences by sex, and correlation and regression analyses were performed to examine the associations between variables of functional capacity (total time on treadmill, perception of balance, and grip strength), quality of life (total score on the FAHI questionnaire), and anthropometric measures (BMI and WHtR). Alpha equal to or less than 0.05 was applied to detect statistical significance. All statistical analyses were conducted using STATA software (version 15.1; STATA Corp. LLC, College Station, TX, USA).

\section{Results}

Table 1 shows the descriptive characteristics of all the study participants and by sex. Figure 1 shows the recruitment process according to the consort flow diagram. The average age of study participants was $58.7 \pm 9.0$ years, with values fluctuating between 40 and 80 years, and no significant difference between men and women was observed. Men and women were also similar $(p>0.05)$ in the years since HIV diagnosis, with an average of about 20 years, and their CD4s were in the normal range. Participants in this study 
visited the gym $2.7 \cdot \pm 1.3 \cdot$ times per week with no significant difference between men and women $(p>0.05)$, and $0.5 \pm 0.5$ have participated in psychosocial support, with no difference between men and women $(p>0.05)$. The results of the $Q O L$ questionnaire between men and women showed no significant differences in the total FAHI score or the physical, emotional, social, and cognitive dimensions of well-being. However, the score on general functioning status was higher in women than in men.

Functional capacity test results between male and female participants showed no significant

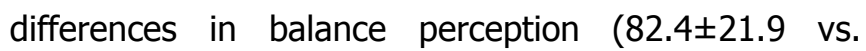

$77.5 \pm 24.4 ; p=0.615)$, total time on the treadmill $(8.9 \pm 5.2$ vs. $8.6 \pm 4.4 \mathrm{~min} ; \mathrm{p}=0.869)$, and VO2 (39.5 \pm 8.1 vs. $35.1 \pm 13.2 ; p=0.413)$. However, as expected, men had greater left and right-hand grip strength compared to women $(86.9 \pm 18.8,56.9 \pm 26.8$, $\mathrm{p}=0.003$; and $87.6 \pm 15.1$, vs. $61.4 \pm 26.6 \mathrm{lb}, \mathrm{p}=0.004$, respectively). Regarding anthropometric measures, other than males being taller than females, no significant difference was observed between men and women in weight, BMI, waist circumference, and WHtR. 'the average BMI of $28.0 \pm 4.9 \mathrm{~kg} / \mathrm{m}^{2}$ classified men and women in the overweight category.

\section{CONSORT 2010 Flow Diagram|}

Enrollment-27

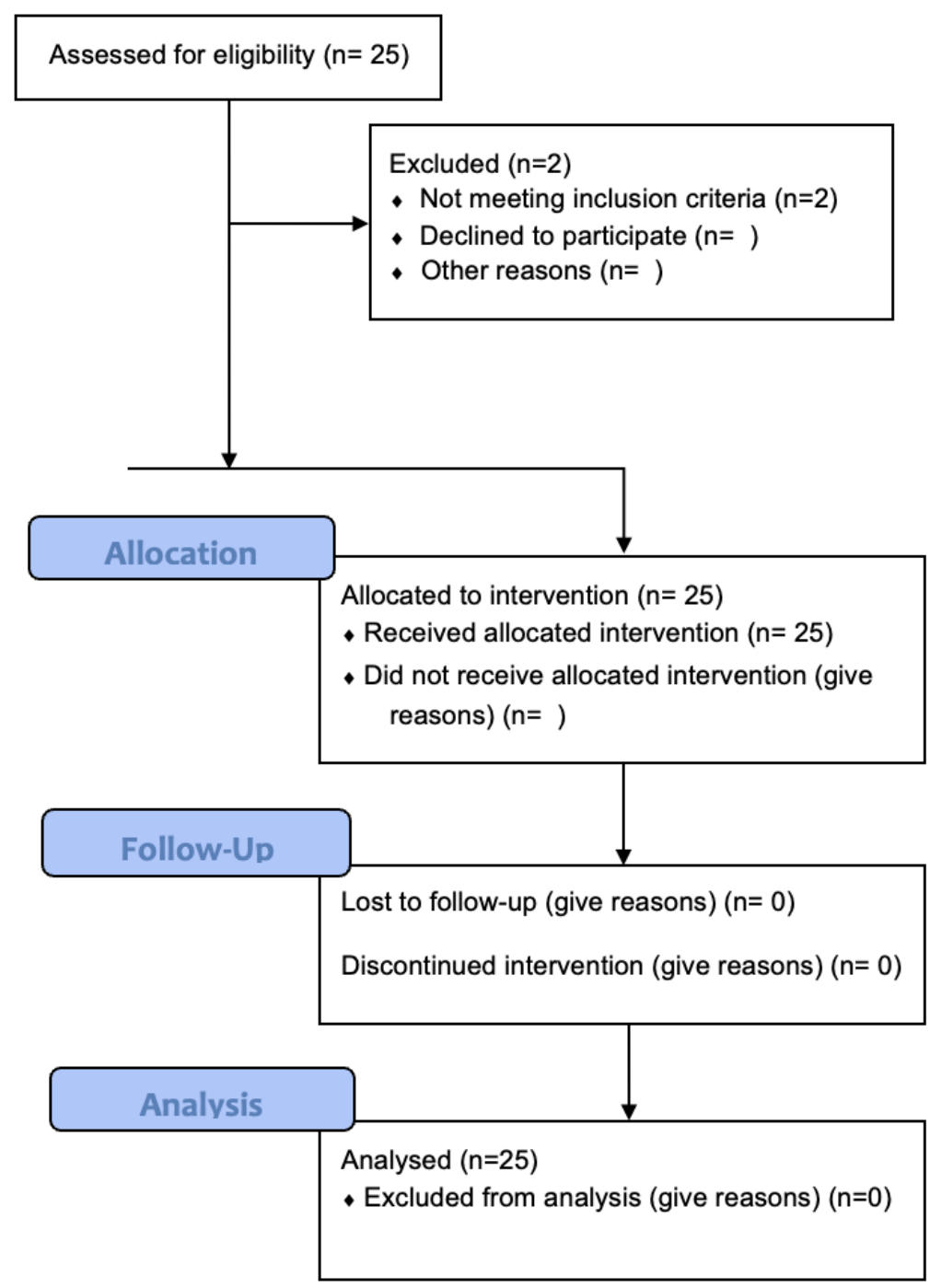

Figure 1. COSORT flow diagram 
Table 1. T-test comparing men and women in all study variables

\begin{tabular}{|c|c|c|c|c|}
\hline General Characteristics & All $(n=25)$ & Men $(n=15)$ & Women $(n=10)$ & $t(p)$ \\
\hline Age (Years) & $58.7 \pm 9.0$ & $59.9 \pm 9.74$ & $56.9 \pm 7.8$ & $0.823(0.419)$ \\
\hline CD4 (Cel mm3) & $762.6 \pm 413.9$ & $769.6 \pm 364.9$ & $860.6 \pm 431.3$ & $0.569(0.575)$ \\
\hline Years of diagnostic & $19.8 \pm 7.2$ & $19.7 \pm 6.7$ & $20 \pm 8.6$ & $-0.089(0.930)$ \\
\hline Gym visits per week & $2.7 \pm 1.3$ & $2.9 \pm 0.9$ & $2.4 \pm 1.7$ & $0.558(0.467)$ \\
\hline Psychosocial Support & $0.5 \pm 0.5$ & $0.5 \pm 0.5$ & $0.6 \pm 0.5$ & $0.075(0.788)$ \\
\hline \multicolumn{5}{|l|}{ Quality of Life } \\
\hline General Physical Health & $13.2 \pm 11.0$ & $14.1 \pm 11.9$ & $11.7 \pm 9.8$ & $0.535(0.598)$ \\
\hline Emotional State & $9.6 \pm 10.5$ & $10.7 \pm 11.0$ & $8 \pm 10.2$ & $0.627(0.537)$ \\
\hline General Status of Functionality & $33.2 \pm 11.8$ & $29.3 \pm 2.8$ & $39.1 \pm 7.4$ & $-2.188(0.039)$ \\
\hline Social Environment & $18.8 \pm 9.8$ & $17.3 \pm 0.7$ & $21 \pm 8.3$ & $-0.929(0.363)$ \\
\hline Mental Activity & $6.3 \pm 2.2$ & $6.4 \pm 2.4$ & $6.2 \pm 1.8$ & $0.221(0.827)$ \\
\hline Total Score & $81.08 \pm 18.4$ & $77.8 \pm 19.3$ & $86 \pm 16.8$ & $-1.095(0.285)$ \\
\hline \multicolumn{5}{|l|}{ Functional Capacity } \\
\hline ABC Scale (\%) & $79.4 \pm 23.1$ & $77.5 \pm 24.4$ & $82.4 \pm 21.9$ & $-0.509(0.615)$ \\
\hline Time on the treadmill (min) & $8.9 \pm 4.8$ & $9.0 \pm 5.3$ & $8.6 \pm 4.4$ & $0.166(0.869)$ \\
\hline Hearth Rate & $132.8 \pm 18.3$ & $130.2 \pm 14.7$ & $136.6 \pm 23.2$ & $0.485(0.497)$ \\
\hline VO2 est. (mL kg min) & $37.7 \pm 10.4$ & $39.5 \pm 8.1$ & $35.1 \pm 13.2$ & $0.710(0.413)$ \\
\hline Left Grip Strength (Ib) & $74.9 \pm 26.5$ & $86.9 \pm 18.8$ & $56.9 \pm 26.8$ & $3.290(0.003)$ \\
\hline Right Grip Strength (lb) & $77.12 \pm 23.9$ & $87.6 \pm 15.1$ & $61.4 \pm 26.6$ & $3.142(0.004)$ \\
\hline \multicolumn{5}{|c|}{ Anthropometric Characteristics } \\
\hline Weight (kg) & $79.2 \pm 15.2$ & $79.2 \pm 13.2$ & $79.0+18.5$ & $0.033(0.974)$ \\
\hline Height (cm) & $168.2 \pm 9.9$ & $172.0 \pm 8.2$ & $162.4 \pm 9.7$ & $2.660(0.014)$ \\
\hline BMI $\left(\mathrm{kg} \mathrm{m}^{-2}\right)$ & $28.0 \pm 4.9$ & $26.8 \pm 4.3$ & $29.8 \pm 5.5$ & $-1.524(0.141)$ \\
\hline WC $(\mathrm{cm})$ & $91.3 \pm 10.8$ & $92.0 \pm 10.5$ & $90.1 \pm 11.8$ & $0.430(0.671)$ \\
\hline WHtR & $0.5 \pm 0.1$ & $0.5 \pm 0.1$ & $0.6 \pm 0.5$ & $-0.772(0.448)$ \\
\hline
\end{tabular}

*Abbreviations: $\mathrm{ABC}=$ Activities-Specific Balance Confidence Scale, BMI=Body Mass Index, WC=Waist Circumference, WHtR=Waist to Height Ratio. 
Table 2. Correlation between functional capacity, anthropometric characteristics, and quality of life

\begin{tabular}{|c|c|c|c|c|c|c|}
\hline Characteristics & Total FAHI r (p) & $\mathrm{R} 2$ & BMI $r(p)$ & R2 & WHtR r (p) & R2 \\
\hline ABC Scale & $-0.236(0.174)$ & 0.0787 & $-0.014(0.839)$ & 0.0018 & $-0.192(0.568)$ & 0.0144 \\
\hline Time on Treadmill & $0.050(0.792)$ & 0.0031 & $0.003(0.807)$ & 0.0026 & $-0.032(0.793)$ & 0.0031 \\
\hline Left Grip Strength & $-0.277(0.935)$ & 0.0003 & $0.002(0.997)$ & 0.0000 & $-0.059(0.545)$ & 0.0162 \\
\hline Right Grip Strength & $-0.266(0.897)$ & 0.0007 & $0.194(0.519)$ & 0.0183 & $0.057(0.798)$ & 0.0029 \\
\hline BMI & $0.343(0.143)$ & 0.0909 & & & & \\
\hline WHtR & $0.379(0.121)$ & 0.1011 & & & & \\
\hline
\end{tabular}

Abbreviations: $A B C=$ Activities-Specific Balance Confidence Scale, $B M I=$ Body Mass Index, WHtR $=$ Waist Circumference and Height Ratio.

Table 3. Correlation between functional capacity and anthropometric characteristics with quality-of-life aspects

\begin{tabular}{|l|l|l|l|l|l|}
\hline Characteristics & $\begin{array}{l}\text { General Physical } \\
\text { Health }\end{array}$ & Emotional State & $\begin{array}{l}\text { General Status of } \\
\text { Functionality }\end{array}$ & $\begin{array}{l}\text { Social } \\
\text { Aspect }\end{array}$ & $\begin{array}{l}\text { Mental } \\
\text { Activity }\end{array}$ \\
\hline ABC Scale & -0.496 & -0.428 & 0.252 & 0.300 & -0.255 \\
\hline Time on treadmill & -0.133 & -0.083 & 0.174 & 0.136 & -0.077 \\
\hline Left Grip Strength & 0.052 & 0.161 & -0.450 & -0.228 & 0.010 \\
\hline Right Grip Strength & 0.095 & 0.219 & -0.475 & -0.268 & -0.071 \\
\hline BMI & 0.4654 & 0.4280 & -0.1244 & -0.1332 & -0.0927 \\
\hline WHtR & 0.6029 & 0.4615 & -0.2335 & -0.1095 & -0.1095 \\
\hline
\end{tabular}

Abbreviations: $\mathrm{ABC}=$ Activities-Specific Balance Confidence Scale, $\mathrm{BMI}=$ Body Mass Index, WHtR= Waist Circumference and Height Ratio.

The waist circumference average in men was $92.0 \pm 10.5 \mathrm{~cm}$, which suggests that obesity is not a risk factor for metabolic and cardiovascular disease. On the other hand, women's average $(90.1 \pm 11.8 \mathrm{~cm})$ suggests central obesity, a risk factor for cardiovascular and metabolic diseases. The participants in this study had an average WHrR of $0.5 \pm 0.1$, which indicates a low health risk by central adiposity compared with the general population. However, with a WHtR of 0.6 , similar to waist circumference, women are considered at risk of central adiposity.
Table 2 shows the correlations between functional capacity variables, anthropometric characteristics, and quality of life in all study participants. The only variables that showed a potential moderate positive tendency with total quality of life score were WHtR $(r=0.38, p=0.12)$ and BMI $(r=0.379$, $p=0.143)$, suggesting that those with higher BMI and WHtR also had higher quality of life scores with the FAHI. Correlations between FAHI segments, functional capacity tests, and anthropometric variables did not reach statistical significance. Table 3 shows the 
correlations between functional capacity variables, anthropometric characteristics, and quality of life dimensions. BMI and WHtR seem to have more effects on general physical health $(r=0.4654, r=0.6029)$ and emotional state $(r=0.4280, r=0.4615)$, respectively.

\section{Discussion}

The primary intention of this investigation was to characterize and assess the relationship between functional capacity variables, anthropometric characteristics, and quality of life in $\mathrm{HIV}+$ patients in Puerto Rico and determine potential differences between men and women. Functional capacity measures raised some health concerns among our study participants. For example, cardiorespiratory fitness assessed with treadmill time using the Ross protocol placed our participants in the poor category [19]. This observation is consistent with other studies on HIV+ Hispanics in Puerto Rico [5,37] and highlights the need to integrate this functional capacity component in clinical evaluations and exercise programs for these patients. Another concern related to those living with HIV is postural instability in asymptomatic individuals [36] \& and the reduced balance perception among this population, increasing the risk of falling [16-17]. Therefore, activities to improve functional capacity are recommended to be added to exercise prescriptions for HIV+ adults as they get older.

The first main finding of this study highlighted the comparability of genders in cardiorespiratory fitness, perception of balance, and functional capacity. Contrary to the current study, gender difference has been previously observed and explained by a more significant muscle mass development, usually due to higher physical activity levels and androgen load in men [20], higher cardiovascular values in males than females [21]. During the aging process, muscle mass typically decreases in both sexes but appears to occur earlier in women than in men [20]. There is evidence associating aging and menopause with loss in muscle mass and musculoskeletal tissue quality among women [22], which provides a potential explanation for our findings. However, average grip strength was comparable with normative data in the general population, classifying our male group in the fair category and females in the good category [23]. It is possible that being enrolled and participating in a structured exercise program, including resistance training, could have influenced the above result.
Therefore, future studies should explore different levels of physical fitness in those living with HIV.

Another noteworthy observation was the significantly higher general functioning status score in females than in males, indicating that HIV+ women self-perceived as more functional and satisfied with their daily activities than HIV+ men. This observation is compatible with Holzemer [24] and Parcesepe [25] but different from those reported by Chandra [26], Kholi [27], and Shor-Posner et al. [28]. In India [26-27] and the US [28], men scored higher than women on positive feelings, sexual activity, financial resources, transport, and environmental control. The authors suggest that as the severity of the disease increases, gender differences accentuate with decreases in positive feelings and financial resources among women. The general functioning score difference between males and females in the referenced studies could also reflect education and social support outside the family nucleus [29].

Nonetheless, this section of the FAHI in our study, particularly among women, could be explained by the psychosocial support provided by the LPGP community-based program. Personal satisfaction and constructive personal care and support can lead to a higher perception of physical and emotional health [30]. However, the psychosocial support attendance data were equivalent between men and women in this study.

Another important observation in this study showed a tendency for anthropometric characteristics to be associated with quality of life. Anthropometric components such as BMI and WHtR were significantly correlated with quality of life, suggesting that lower BMI and lower WHtR are likely to have a lower total quality of life score. This unexpected observation contradicts the previously mentioned by Mbada [31] that pointed functional capacity and adequate anthropometric variables could be recognized as good predictors of quality of life. Similarly, the research evidence shows lower quality of life scores in underweight and obese adults compared to average weight and overweight adults [32]. Future endeavors should explore these bodyweight factors in more detail in the same population, Hispanic-Latinx.

Previous research has mentioned the benefits of physical exercise outcomes associated with quality of life and immune system in the HIV+ community $[6,17,33]$. In addition, mental health [34], social support [30], and spirituality [35] influence the quality 
of life among HIV-positive adults, thereby potentially affecting the associations between functional capacity and quality of life. Amongst the variety of services in the LPGP community-based program, patients can participate in social activities, spiritual gatherings and seek professional mental health. Future studies should also focus on social interaction time, spiritual interactions through prayers, conversations with spiritual leaders, and mental health status as potential confounders of the association between functional capacity and quality of life in HIV+ adults in Puerto Rico.

\section{Conclusion}

The current study characterized aspects of functional capacity, anthropometry, and quality of life among HIV+ Hispanics participating in a communitybased exercise program in Puerto Rico. Although functional status by self-report was higher among women, no significant associations were detected between functional capacity, anthropometric variables, and quality of life. However, a tendency for high BMI and WHtR among those with lower QOL scores might support the need to integrate these assessments as potential health outcomes in exercise programs for HIV+ adults. Finally, high waist circumference and WHtR among HIV+ women are a concern that must be addressed in HIV clinics and exercise programs. In addition, future studies should delve into the impact of motor components, such as gait speed and posture, in association with the various factors the current study highlighted to better comprehend the impact of HIV on quality of life in this population.

\section{References}

[1] US Department of Health and Human Services, HIV.gov (2019) Global Statistics. Retrieved February 6, 2020, from https://www.hiv.gov/hiv-basics/overview/dataand-trends/global-statistics

[2] US Department of Health and Human Services, HIV.gov (2019) Impact on Racial and Ethnic Minorities. Retrieved February 6, 2020, from https://www.hiv.gov/hiv-basics/overview/dataand-trends/impact-on-racial-and-ethnicminorities

[3] Diagnoses of HIV Infection in the United States and Dependent Areas, (2017), HIV Surveillance Report, 1-129.
[4] F. A. Ramírez-Marrero, E. De Jesús, J. Santana-Bagur, R. Hunter, W. Frontera, M. J. Joyner, Prevalence of cardiometabolic risk factors in Hispanics living with HIV, Ethnicity \& disease, 20 (2010) 423-428. [PubMed]

[5] F. A. Ramírez-Marrero, J. L. Santana-Bagur, M. J. Joyner, J. Rodríguez-Zayas, W. Frontera, Metabolic syndrome in relation to cardiorespiratory fitness, active and sedentary behavior in HIV+ Hispanics with and without lipodystrophy, Puerto Rico health sciences journal, 33 (2014) 163-169. [PubMed]

[6] N.N. Quiles, M. Rosario, A. Ortiz, Balance as an assessment of health-related quality of life in people living with HIV, Journal of Human Sport and Exercise, 14 (2019) 492-499. [DOI]

[7] L. O. Bauer, N.A. Ceballos, S J. D. hanley, L.I. Wolfson, Sensorimotor Dysfunction in HIV/AIDS: Effects of Antiretroviral Treatment and Comorbid Psychiatric Disorders, Aids, 19 (2005) 495-502. [DOI] [PubMed]

[8] M.G. Rosario, J. Marshall, A. Herkert, B. Binoy, H. Windham, E. Orozco, Lower Limb Neuromuscular Modification and Standing Postural Control Alteration in Apparent Asymptomatic People Living with HIV, ournal of Rehabilitation Practices and Research, 1 (2020) 1-9. [DOI]

[9] L. Richert, P. Dehail, P. Mercié, F.-A. Dauchy, M. Bruyand, C. Greib, G. Chêne, Groupe d'Epidémiologie Clinique du SIDA en Aquitaine (GECSA), High frequency of poor locomotor performance in HIV-infected patients, Aids, 25 (2011) 797-805. [DOI] [PubMed]

[10] K. Oursler, J. Sorkin, B. Smith, L. Katzel, Reduced Aerobic Capacity and Physical Functioning in Older HIV-Infected Men, AIDS Research and Human Retroviruses, 22 (2006) 1113-1121. [DOI] [PubMed]

[11] S. Garland, P. Ingesson, H. Petersson, A. Wisén, Is Ross treadmill method an alternative to Åstrand cycle ergometer method?, European Journal of Physiotherapy, 19 (2017) 167-172. [DOI]

[12] J.T. Ciccolo, E.M. Jowers, J. B. Bartholomew, The Benefits of Exercise Training for Quality of Life in HIV/AIDS in the Post-HAART Era, Sports Medicine, 34 (2004) 487-499. [DOI] [PubMed] 
[13] S. Ramlagan, K. Peltzer, N. Phaswana-Mafuya, Handgrip strength and associated factors in non-institutionalised men and women 50 years and older in South Africa, BMC Research Notes, 7 (2014) 1-7. [DOI] [PubMed]

[14] Luna Heredia, E., Martin Peña, G., \& Ruiz Galiana, J. Handgrip dynamometry in healthy adults, Clinical Nutrition, 24 (2005) 250-258. [DOI] [PubMed]

[15] A.L. Khoury, M.M. C.orey, T.C. Wong, D.L. Mcneil, B. Humphries, K. Frankey, M.S. Mckellar, Diminished physical function in older HIV-infected adults in the Southeastern US despite successful antiretroviral therapy, PLoS One, 12 (2017) e0179874. [DOI] [PubMed]

[16] Y. Lajoie, S. Gallagher, Predicting falls within the elderly community: comparison of postural sway, reaction time, the Berg balance scale and the Activities-specific Balance Confidence $(A B C)$ scale for comparing fallers and nonfallers, Archives of Gerontology and Geriatrics, 38 (2004) 11-26. [DOI] [PubMed]

[17] M.G. Rosario, Early signs of standing postural instability in asymptomatic people living with HIV. HIV \& AIDS Review, International Journal of HIV-Related Problems, 219 (2020) 193-198. [DOI]

[18] M. Viala-Danten, D. Dubois, H. Gilet, S. Martin, K. Peeters, D. Cella, Psychometric evaluation of the functional assessment of HIV Infection (FAHI) questionnaire and its usefulness in clinical trials, Quality of Life Research, 19 (2010) 1215-1227. [DOI] [PubMed]

[19] L.S. Pescatello, D. Riebe, P. D. Thompson, (2018) ACSM's guidelines for exercise testing and prescription, Lippincott Williams \& Wilkins.

[20] S.E. Mancilla, F. S. Ramos, B. P. Morales, Association between handgrip strength and functional performance in Chilean older people, Revista Medical de Chile, 144 (2016) 598-603. [DOI] [PubMed]

[21] M.G. Rosario, L. Jamison, G. Gines, Motor and Cardiovascular distinctions among genders in Hispanic-Latinx people living with HIV, International Journal of Physiotherapy, 8 (2021) 64-69. [DOI]

[22] R.P.H.A. onkainen, V. Kovanen, M. Alén, E. Pöllänen, E.-M. Palonen, C. Ankarberg-
Lindgren, S. Sipilä, Postmenopausal hormone replacement therapy modifies skeletal muscle composition and function: a study with monozygotic twin pairs, Journal of Applied Physiology, 107 (2009) 25-33. [DOI] [PubMed]

[23] V. Heyward, A. Gibson, (2014) Advanced Fitness Assessment and Exercise Prescription, Seventh edition, Human Kinetics, 1-552.

[24] W.L. Holzemer, J.G. Spicer, H.S. Wilson, J.K. Kemppainen, C. Coleman, Validation of the quality of life scale: living with HIV, Journal of Advanced Nursing, 28 (1998) 622-630. [DOI] [PubMed]

[25] A.M. Parcesepe, D. Nash, O. Tymejczyk, W. Reidy, S.G. Kulkarni, B. Elul, HIV-Related Stigma, and Health-Related Quality of Life Among Adults Enrolling in HIV Care in Tanzania, AIDS Behavior,24 (2020) 142-150. [DOI] [PubMed]

[26] P.S. Chandra, V.A. Satyanarayana, P. Satishchandra, K.S. Satish, M. Kumar, Do Men and Women with HIV Differ in Their Quality of Life? A Study from South India, AIDS Behavior, 13 (2009) 110-117. [DOI] [PubMed]

[27] R.M. Kohli, S. Sane, K. Kumar, R.S. Paranjape, S.M. Mehendale, Assessment of quality of life among HIV-infected persons in Pune, India, Quality of Life Research, 14 (2005) 16411647. [DOI] [PubMed]

[28] G. Shor-Posner, R. Lecusay, M.J. MiquezBurbano, J. Quesada, A. Rodriquez, P. Ruiz, S O'Mellan, A Campa, H. Rincon, F. Wilkie, J B Page, M.K. Baum, Quality of Life measures in the Miami HIV-1 infected drug abusers cohort: Relationship to gender and disease status, Journal of Substance Abuse, 11 (2000) 395404. [DOI] [PubMed]

[29] A. Tesfay, A. Gebremariam, M, Gerbaba, H. Abrha, Gender differences in health-related quality of life among people living with HIV on highly Active antiretroviral therapy in Mekelle Town, Northern Ethiopia, BioMed Research International, (2015) 1-9. [DOI] [PubMed]

[30] S. Swindells, J. Mohr, J. C. Justis, S. Berman, C. Squier, M.M. Wagener, N. Singh, Quality of life in patients with human immunodeficiency virus infection: impact of social support, coping style and hopelessness, International Journal 
of STD \& AIDS, 10 (1999) 383-391. [DOI] [PubMed]

[31] C.E. Mbada, O. Onayemi, Y. Ogunmoyole, O. Johnson, C.O. Akosile, Health-related quality of life and physical functioning in people living with HIV/AIDS: a case-control design, Health and Quality of Life Outcomes, 11 (2013) 1-8. [DOI] [PubMed]

[32] S. George, C. Bergin, S. Clarke, G. Courtney, Mary B Codd, Health-related quality of life and associated factors in people with HIV: an Irish cohort study, Health Qual Life Outcomes 14 (2016) 1-12. [DOI] [PubMed]

[33] E. Orozco, M.G. Rosario, Overall fitness benefits in individuals with HIV participating in a community-based exercise program, Journal of Rehabilitation Practices and Research, 1 (2020) 1-5. [DOI]

[34] A. Douaihy, N. Singh, Factors affecting quality of life in patients with HIV infection, The AIDS Reader, 11 (2001) 450-454. [PubMed]

[35] K.B. Grill, J. Wang, Y.I. Cheng, M.E. Lyon, The role of religiousness and spirituality in healthrelated quality of life of persons living with HIV: A latent class analysis, Psychology of Religion and Spirituality, 12 (2020) 494-504. [DOI] [PubMed]

[36] M.G. Rosario, A. Schacherl, G. Bevers, K. Longoria, A. Versemann, Ankle complex Neuromuscular Coordination variation in people living with HIV, International Journal of Physiotherapy, 8 (2021) 52-58. [DOI]

[37] F.A. Ramírez-Marrero, V. Wojna, J. SantanaBagur, W.R. Frontera, Cardiorespiratory Fitness Relative to Lean Body Mass in HIV and HIVWomen, Journal of Clinical Exercise Physiology, 8 (2019) 138-143. [DOI]
Funding

No funding was received for conducting this study.

\section{Authors contribution}

All authors contributed to the study's conception and design

\section{Ethics Approval}

IRB approval TWU protocol \# 20091

\section{Informed consent}

The participant gave signed consent for this study

\section{Conflict of interest}

The Authors have no conflicts of interest to declare that they are relevant to the content of this article.

\section{Does this article screened for similarity Yes}

\section{About The License}

(c) The Author(s) 2021. The text of this article is open access and licensed under a Creative Commons Attribution 4.0 International License 